\title{
Differential Co-Occurring Patterns Between Depressive Symptomatology and Sleep-Wake- Related Disturbances in Mood Disorders
}

\author{
Sze-Yu Chen' \\ Chun-Hao Chen' \\ Chen Lo' \\ Mong-Liang $\mathrm{Lu}^{2}$ \\ Cheng-Dien $\mathrm{Hsu}^{3}$ \\ Yi-Hang Chiu ${ }^{2}$ \\ Chun-Hsin Chen ${ }^{2}$ \\ Shih-Ku Lin ${ }^{4,5}$ \\ Ming-Chyi Huang ${ }^{5}$ \\ Hsi-Chung Chen (DI) \\ Po-Hsiu Kuo ${ }^{1,7}$ \\ 'Department of Psychiatry, National \\ Taiwan University Hospital, Taipei, \\ Taiwan; ${ }^{2}$ Department of Psychiatry, Wan- \\ Fang Hospital \& School of Medicine, \\ College of Medicine, Taipei Medical \\ University, Taipei, Taiwan; ${ }^{3}$ Department \\ of Psychosomatic Medicine, Taiwan \\ Adventist Hospital, Taipei, Taiwan; \\ ${ }^{4}$ Department of Psychiatry, Chang Gung \\ Memorial Hospital at Linkou, Taoyuan, \\ Taiwan; ${ }^{5}$ Department of Psychiatry, Taipei \\ City Hospital, Songde Branch, Taipei, \\ Taiwan; ${ }^{6}$ Center of Sleep Disorders, \\ National Taiwan University Hospital, \\ Taipei, Taiwan; ${ }^{7}$ Institute of Epidemiology \\ and Preventive Medicine, College of \\ Public Health, National Taiwan University, \\ Taipei, Taiwan
}

Correspondence: Hsi-Chung Chen Email hsichungchen@ntu.edu.tw
Purpose: This study aimed to elucidate the various co-occurring patterns of depressive symptomatology and sleep-wake-related disturbances (SWRDs) in patients with mood disorders.

Patients and Methods: Individuals in non-acute states of major depressive disorder or bipolar disorder were recruited. The Beck Depression Inventory II (BDI-II) was utilized to evaluate depressive symptoms. BDI-II items were classified into three domains: cognitive, affective, and somatic. Between-domain differences with various SWRDs were examined. Latent class analysis was used to empirically classify participants using BDI-II items as indicator variables. Co-occurring patterns between domains of BDI-II items and SWRDs were re-examined in each subgroup to elucidate inter-individual differences.

Results: In total, 657 participants were enrolled. Of participants, $66.8 \%$ were female, and $52.4 \%$ were diagnosed with major depressive disorder. Each BDI-II domain exhibited different co-occurring patterns. The somatic domain was most likely to co-occur with various SWRDs. Three subgroups were derived from latent class analysis and were designated as poor sleep quality and high insomnia $(n=150)$, poor sleep quality and moderate insomnia $(n=248)$, and poor sleep quality and low insomnia $(n=159)$. The group with more severe insomnia presented with more severe depressive and anxiety symptoms. The three subgroups further differed in co-occurring patterns. From the low insomnia to high insomnia group, the associations with various SWRDs appeared in the sequence of somatic, affective, and cognitive domains.

Conclusion: Co-occurring patterns between domains of depressive symptomatology with various SWRDs differ and may vary among individuals.

Keywords: co-occurring, latent class analysis, mood disorders, sleep-wake-related disturbance, depression

\section{Introduction}

Mood disorders are highly prevalent. ${ }^{1}$ Of the various mood disorders, depression exhibits the highest recurrence and is characterized by chronicity. In patients with bipolar disorder, depression, rather than mania, comprises a longer duration in the disease course, ${ }^{2}$ and depressive symptoms have the greatest impact on quality of life. $^{3}$ Sleep-wake-related disturbances (SWRDs) are strongly correlated with depression. More than $80 \%$ of patients with depression experience prominent insomnia. ${ }^{4}$ Further, sleep architecture in individuals with depression differs from that of healthy controls, even during the remission state. ${ }^{5}$ The bidirectional relationship between depression and SWRDs has been established. Depression may 
precede complaints of insomnia, ${ }^{6}$ and insomnia may predict the new onset $^{7}$ or recurrence of depression. ${ }^{7-9}$ In summary, SWRDs are considered a core symptom of depression and serve as an important factor when investigating the neurobiological mechanisms of depression.

Various neural circuits underpin depression ${ }^{10}$ and SWRDs. ${ }^{11}$ The neurobiology of sleep-wake regulation overlaps considerably with that of mood. ${ }^{12,13}$ For instance, patients with depression exhibited increased rapid eye movement (REM) sleep; this phenomenon was amplified when cholinergic neurotransmission was augmented. Healthy participants administered with cholinomimetics developed depressive symptoms including anhedonia and fatigue. ${ }^{5}$ Further, most antidepressants alter sleep architecture. Administration of selective serotonin reuptake inhibitors or monoamine oxidase inhibitors decreased the total duration of REM sleep, and a transient decrement in serotonin levels achieved by tryptophan depletion reversed this effect. ${ }^{14}$ Electroconvulsive therapy has consistently been shown to lengthen sleep duration, improve sleep efficacy, and shift REM sleep patterns to approximate those of healthy sleepers. ${ }^{15}$ In summary, depressive symptomatology and various SWRDs are closely related and may share common neurobiological pathomechanisms. An in-depth exploration of their specific co-occurring relationships may reveal shared biophysical vulnerabilities and address issues arising from the heterogeneity of depression.

Common psychometrics that are used to measure depressive symptomatology include over 50 depressive symptoms. ${ }^{16}$ Thus, each domain of depression may possess diverse associations with specific SWRDs. A recent network analysis revealed different associations between the items of the Inventory of Depressive Symptomatology with insomnia. Specifically, depression was partially associated with insomnia, but irritability, anxiety, and interpersonal hypersensitivity were not directly associated with insomnia. ${ }^{17}$ Additionally, symptom profiles differ substantially between individuals diagnosed with depressive disorders. ${ }^{18}$ This phenotypic heterogeneity reflects the complex pathogenesis of depression, which hinders precise elucidation of the pathomechanisms of depression and development of precision medicine. ${ }^{19}$ Consequently, when investigating the associations between depressive symptomatology and SWRDs, individual differences in depressive symptomatology should be considered.

Thus, in the present study, the associations between depressive symptomatology and SWRDs were examined in participants diagnosed with mood disorders. First, the independent associations between each fundamental domain of depression (cognitive, affective, and somatic) and SWRDs were examined. Subsequently, with depressive symptoms as indicator variables, latent class analysis (LCA) was utilized to empirically subgroup participants. The associations between SWRDs and depressive domains were then re-examined in participants of each subgroup to elucidate individual differences.

\section{Patients and Methods}

\section{Participants}

Participants were enrolled in three general hospitals and one psychiatric hospital in Taiwan between 2013 and 2018. Individuals fulfilling all the following criteria were referred by clinicians: (a) aged between 18 and 65 years old; (b) diagnosed with major depressive disorder (MDD) or bipolar affective disorder (BAD) according to the Diagnostic and Statistical Manual of Mental Disorders (DSM) - IV; and (c) non-acute status, defined as a score less than 17 on the Hamilton Depression Rating Scale ${ }^{20}$ and less than 20 on the Young Mania Rating Scale. ${ }^{21}$ The diagnosis of MDD or BAD in eligible participants was further confirmed using the Chinese version of the Schedule for Affective Disorder and SchizophreniaLifetime (SADS-L). ${ }^{22,23}$ Exclusion criteria included comorbid schizophrenia, intellectual disability, and substance use disorder. Trained interviewers collected comprehensive data on sociodemographic variables (sex, age, marital status, birth location, academic attainment, employment status, and income), lifestyle factors (body mass index, exercise habit, and use of alcohol or tobacco), and history of physical illness and psychiatric conditions (psychiatric diagnosis, hypnotic use, depressive symptoms, and anxiety symptoms). This study was approved by the Institutional Review Board of the National Taiwan University Hospital (N0. 201212134RINB, 201512086RIND, and 202007094RINA). Written informed consent was obtained from each participant, and the study was conducted in accordance with the 2008 revision of the Helsinki Declaration of 1975.

\section{Measurement and Categorization of Depressive Symptomatology}

The Chinese version of the Beck Depression Inventory II (BDI-II) was used to measure and quantify depressive symptoms. The reliability and validity of the Chinese 
version of BDI-II was examined. ${ }^{24}$ In terms of reliability, the Chinese version of the BDI-II has a Cronbach's alpha of 0.94 , and a split-half reliability of 0.91 . Two factors were explored in the exploratory factor analysis, including the cognitive-affective and somatic dimensions. The Chinese version of the BDI-II correlates well with the Chinese Health Questionnaire $(\mathrm{r}=0.69, \mathrm{p}<0.001)$, which indicates an acceptable degree of convergent validity. The criterion validity for depressive disorders, which was measured using the area under the receiver operating characteristic curve, was $0.78 \pm 0.04$, with an optimal cutoff of $16 / 17$, sensitivity of $80.4 \%$, specificity of $62.8 \%$, and positive predictive value of $72.8 \%$. BDI-II included 21 items: sadness (D1), pessimism (D2), past failure (D3), loss of pleasure (D4), guilty feelings (D5), punishment feelings (D6), self-dislike (D7), self-criticalness (D8), suicidal thoughts or wishes (D9), crying (D10), agitation (D11), loss of interest (D12), indecisiveness (D13), worthlessness (D14), loss of energy (D15), changes in sleep patterns (D16), irritability (D17), changes in appetite (D18), concentration difficulties (D19), tiredness or fatigue (D20), and loss of interest in sex (D21). As we aimed to investigate the associations between depressive symptoms and SWRDs, item D16 ("change in sleep patterns") was excluded to prevent the introduction of statistical collinearity. For more relevant clinical implications, the remaining 20 items were divided into three domains based on previous literature, ${ }^{25-27}$ comprising the affective domain (D1, D4, D10, D11, D12, and D17), cognitive domain (D2, D3, D5, D6, D7, D8, D9, D13, D14, and D19), and somatic domain (D15, D18, D20, and D21), respectively. The scores of items in each domain were summed, and their respective associations with various SWRDs were examined.

\section{Measurement of Sleep-Wake-Related Symptoms \\ Sleep Quality}

The Pittsburgh Sleep Quality Index (PSQI) was used to measure sleep quality over the past 1 month. The PSQI comprised 19 items which encompassed seven components, including subjective sleep quality (component 1 , C1), sleep onset latency (component 2, C2), total sleep duration (component 3, C3), sleep efficiency (component 4, C4), sleep disturbances (component 5, C5), use of sleep medication (component 6, C6), and daytime dysfunction (component 7, C7.) Each component was assigned a score between 0 and 3, resulting in a global score of $21 .^{28}$ The Chinese version of the PSQI has been validated, whereby a score of 6 or more indicates poor sleep quality. ${ }^{29}$ Because hypnotic use would confound the association between depressive symptomatology with SWRD, C6 was specified as a covariate in subsequent stratified analyses with each component score. A component score other than zero was defined such "case" in the related analyses.

\section{Chronotype Preference}

Chronotype preference was assessed using the Morningness-Eveningness questionnaire (MEQ). The MEQ is a self-report questionnaire with a total score between 13 and 55 points. Lower scores indicate a preference towards eveningness. ${ }^{30}$ The validity and reliability of the Chinese version of the MEQ have been established. $^{31}$ The lowest quartile of MEQ scores was defined as eveningness preference in the present study.

\section{Night and Daytime Symptoms of Insomnia}

Sleep loss was defined as the gap between actual sleep duration and subjective evaluation of the required sleep duration for each individual. "Sleep loss" was considered a case of actual sleep duration which was 2 or more hours less than the sleep duration subjectively required by an individual. Three questions were adopted to evaluate night symptoms of insomnia: ${ }^{22}$ "In the past 12 months, it takes more than 2 hours almost every night to fall asleep" for sleep initiation difficulty, "Sleep interruption occurs almost every night, and it takes more than one hour to fall asleep again" for sleep maintenance difficulty, and "Being awake more than 2 hours earlier than one expected" for early morning awakening. Daytime symptoms of insomnia were evaluated using the question: "Having persistent daytime sleepiness for at least 2 weeks." Positive responses to the aforementioned questions were defined as "cases" for respective symptoms.

\section{Other Physical and Mental Comorbidities}

History of physical illnesses was defined based on selfreports by the participants, including cardiovascular, endocrine, metabolic, gastrointestinal, urinary, hematological, infectious, neurological/musculoskeletal, and immune/ asthma/allergic diseases. Anxiety symptoms were assessed using the Chinese version of the Beck Anxiety Inventory (BAI). ${ }^{32,33}$ 


\section{Statistical Analyses}

SPSS for Windows, version 17.0 (SPSS, Chicago, IL) was used for most analyses. Mplus version 7 (Muthen \& Muthen) was used for LCA. ${ }^{34}$ Univariate analysis was performed using a $\chi^{2}$ test or analysis of variance (ANOVA). Multivariable regression was used to examine the independent relationships between each depressive domain and SWRDs. The results were expressed as odds ratios (ORs) and 95\% confidence. Moreover, the scores of each domain of the BDI-II, age, sex, BAI scores, psychiatric diagnosis, and hypnotic use were also specified in each logistic regression model to control for the confounding effects. P-values less than 0.05 were considered statistically significant.

\section{Subgrouping by Latent Class Analysis}

To investigate individual differences in the associations between depression domains and SWRDs, participants were empirically subgrouped by LCA. The 20 items of the BDI-II were used as indicator variables for LCA. We further conducted a subgroup-stratified analysis. The optimal number of subgroups was determined using five fitness indices for LCA. Lower values resulted from Akaike's information criterion (AIC), ${ }^{35}$ and sample sizeadjusted Bayesian information criterion (sBIC) $)^{36}$ indicated superior model fits. The value of entropy close to one suggested a clear classification, ${ }^{37}$ while a value higher than 0.7 was considered adequate. ${ }^{38}$ Likelihood ratio tests were implemented to compare fitness between two models. Lo-Mendell-Rubin adjusted likelihood ratio test and parametric bootstrapped likelihood ratio test ${ }^{39}$ were used for comparison between adjacent classes. A significant $p$-value was interpreted as a $\mathrm{k}$ class model with a superior fit to that of k-1 class model. ${ }^{39}$

\section{Results}

In total, 657 participants were enrolled in the present study. Of participants, $66.8 \%$ were female, and $52.7 \%$ were over 45 years old. Of participants, $52.4 \%$ were diagnosed with MDD, and $75.7 \%$ used hypnotics. The average BDI-II score was 14.8 (Standard deviation $[\mathrm{SD}]=11.8$ ). The average BAI score was $13.2(\mathrm{SD}=12.2)$ (Table 1).

Table 2 includes the five fitness indices for LCA models. Based on AIC and sBIC, a five-group model was superior to a four-group model or a three-group model. A three-group model had an entropy value closest to one. According to Lo-Mendell-Rubin adjusted likelihood ratio test, having five subgroups was not superior to having four groups. In both three-group model and four-group model, the major between-group difference was the mean score of BDI, which indicated different severity of depression. Because the homogeneous distribution pattern among the indicator variables and the consideration of statistical power, a three-group was favored in terms of practical utility (Figure S1). Overall, a three-group model had the best entropy value and better clinical utility; therefore, the three-group model was selected. The three subgroups were designated based on their SWRD characteristics: poor sleep quality and high insomnia (PSQ+HI, $\mathrm{n}=150$ ), poor sleep quality and moderate insomnia (PSQ+MI, n=248), and poor sleep quality and low insomnia (PSQ+LI, $\mathrm{n}=159$ ). All three groups were termed as "poor sleep quality" to highlight that poor sleep quality was consistently prevalent across the subgroups (76.7-93.7\%), which was discordant with the relatively low proportion of individuals with insomnia symptoms.

With regard to baseline characteristics, the PSQ+HI group comprised a higher proportion of females (79.3\%), and the PSQ+LI group comprised a higher proportion of individuals with high income $(31.8 \%)$. With regard to lifestyle factors, the PSQ+LI group comprised a higher proportion of participants participating in regular exercise (62.5\%), and the PSQ+MI group comprised the least regular drinkers (11.4\%). The PSQ+HI group exhibited substantially more physical comorbidities and comprised significantly more participants diagnosed with MDD and participants with hypnotics use. The PSQ+HI group exhibited the highest average BDI-II and BAI scores (Table 1).

Table 3 summarizes the SWRD profiles of all participants and the three subgroups. Of participants, $84.9 \%$ had poor sleep quality (PSQI total score $\geq 6$ ), and $34.3 \%$ had subjective sleep loss. Each night and daytime symptom of insomnia was present in approximately one third of participants (30.3-41.0\%). Between-group comparisons revealed that SWRDs were most prevalent in the PSQ $+\mathrm{HI}$ group, slightly less in the PSQ+MI group, and were least prevalent in the PSQ+ LI group with the exception of early morning awakening and daytime sleepiness, which were distinctly more common in the PSQ+LI group than in the PSQ+MI group. Similarly, PSQI-defined poor sleep quality was evident across subgroups, ranging from $76.7 \%$ to $93.7 \%$. Night symptoms of insomnia were lowest in the PSQ+LI group (26.9-29.0\%), but daytime sleepiness was not lowest in the PSQ+LI group (43.8\%).

Figure 1 illustrates the independent relationship between domains of depressive symptomatology and 
Table I Sociodemographic Characteristics of Participants $(n=657)$

\begin{tabular}{|c|c|c|c|c|c|}
\hline & Total & PSQ+HI* & PSQ+MI & PSQ+LI & \multirow[t]{2}{*}{$p$-value for $\chi^{2}$ IANOVA } \\
\hline & $n=657$ & $n=150$ & $n=248$ & $n=259$ & \\
\hline \multicolumn{6}{|l|}{$\operatorname{Sex}(n, \%)$} \\
\hline Female & $439(66.8)$ & $119(79.3)$ & $149(60.1)$ & $175(66.0)$ & $<0.001$ \\
\hline Male & $218(33.2)$ & $31(20.7)$ & 99 (39.9) & $90(34.0)$ & \\
\hline \multicolumn{6}{|l|}{ Age (n, \%) } \\
\hline$\leq 30$ years & $92(15.9)$ & $22(17.1)$ & $33(15.4)$ & $37(15.8)$ & \\
\hline $3 \mathrm{I}-44$ years & $|8|(3 \mid .4)$ & $49(38.0)$ & 71 (33.2) & $61(26.1)$ & 0.13 \\
\hline$\geq 45$ years & $304(52.7)$ & $58(45.0)$ & $1 \mathrm{I} 0(5 \mathrm{I} .4)$ & $136(58.1)$ & \\
\hline \multicolumn{6}{|l|}{ Marital status (n, \%) } \\
\hline Single & $217(37.6)$ & $54(41.9)$ & $80(37.4)$ & $83(35.5)$ & 0.06 \\
\hline Married & $25 I(43.5)$ & $43(33.3)$ & $94(43.9)$ & II 4 (48.7) & \\
\hline Other & $109(18.9)$ & $32(24.8)$ & $40(18.7)$ & $37(15.8)$ & \\
\hline \multicolumn{6}{|l|}{ Birth place (n, \%) } \\
\hline Urban & $450(79.8)$ & III (86.7) & 159 (75.7) & $180(79.6)$ & 0.05 \\
\hline Rural & II4 (20.2) & $17(13.3)$ & $51(24.3)$ & $46(20.4)$ & \\
\hline \multicolumn{6}{|l|}{ Education (n, \%) } \\
\hline University and higher & $27 I(47.1)$ & $48(37.5)$ & $112(52.3)$ & III (47.6) & \\
\hline Senior high school & $205(35.7)$ & $53(4 I .4)$ & $72(33.6)$ & $80(34.3)$ & 0.10 \\
\hline Junior high school and lower & $99(17.2)$ & $27(21.1)$ & $30(14.0)$ & $42(18.0)$ & \\
\hline \multicolumn{6}{|l|}{ Work status (n, \%) } \\
\hline Employed & $221(38.6)$ & $46(35.7)$ & $83(39.3)$ & $92(39.5)$ & 0.74 \\
\hline Unemployed & $352(61.4)$ & $83(64.3)$ & $128(60.7)$ & $|4|(60.5)$ & \\
\hline \multicolumn{6}{|l|}{ Income (n, \%) } \\
\hline High ( $\geq 40,000$ NTD/month) & $157(28.6)$ & $23(19.0)$ & $63(30.7)$ & $71(31.8)$ & \\
\hline Moderate (10,000-39,999 NTD/month) & $174(3 \mid .7)$ & $45(37.2)$ & $55(26.8)$ & $74(33.2)$ & 0.04 \\
\hline Low (0-9999 NTD/month) & $218(39.7)$ & $53(43.8)$ & $87(42.4)$ & $78(35.0)$ & \\
\hline \multicolumn{6}{|l|}{ Body mass index $(n, \%)$} \\
\hline Healthy weight $\left(18.5-<24.0 \mathrm{~kg} / \mathrm{m}^{2}\right)$ & $305(54.0)$ & $63(50.0)$ & III (53.I) & $|3|(57.0)$ & \\
\hline Underweight $\left(<18.5 \mathrm{~kg} / \mathrm{m}^{2}\right)$ & $43(7.6)$ & II (8.7) & $19(9.1)$ & $13(5.7)$ & 0.61 \\
\hline Overweight $\left(24.0-<27.0 \mathrm{~kg} / \mathrm{m}^{2}\right)$ & $155(27.4)$ & $34(27.0)$ & $57(27.3)$ & $64(27.8)$ & \\
\hline Obesity $\left(\geq 27.0 \mathrm{~kg} / \mathrm{m}^{2}\right)$ & $62(11.0)$ & $18(14.3)$ & $22(10.5)$ & $22(9.6)$ & \\
\hline \multicolumn{6}{|l|}{ Lifestyle (n, \%) } \\
\hline Regular exercise (> 2 times/week) & $312(54.7)$ & $52(40.6)$ & II $5(54.8)$ & $145(62.5)$ & $<0.001$ \\
\hline \multicolumn{6}{|l|}{ Cigarette use } \\
\hline Non-smoker & $372(65.7)$ & $81(64.8)$ & $136(64.2)$ & $155(67.7)$ & \\
\hline Ex-smoker & $75(13.3)$ & $12(9.6)$ & $32(15.1)$ & $31(13.5)$ & 0.43 \\
\hline Current smoker & $119(21.0)$ & $32(25.6)$ & $44(20.8)$ & $43(18.8)$ & \\
\hline Regular alcohol drinking $>$ lyear & $96(17.1)$ & $27(21.8)$ & $26(11.4)$ & $43(20.5)$ & 0.01 \\
\hline \multicolumn{6}{|l|}{ Comorbid disease (n, \%) } \\
\hline Cardiovascular disease & $132(23.2)$ & $23(18.0)$ & $51(24.2)$ & $58(25.1)$ & 0.28 \\
\hline Endocrinology and metabolism disease & $164(29.1)$ & $45(35.4)$ & $51(24.4)$ & $68(30.0)$ & 0.10 \\
\hline Gastroenterology disease & $260(45.5)$ & $78(60.9)$ & $97(45.8)$ & $85(36.6)$ & $<0.001$ \\
\hline Urinary system disease & $62(10.9)$ & $17(13.3)$ & $29(13.8)$ & $16(6.9)$ & 0.04 \\
\hline Infectious disease & $9(1.6)$ & I (0.8) & $5(2.4)$ & $3(1.3)$ & 0.47 \\
\hline Neuromuscular disease & $201(35.3)$ & $62(48.8)$ & $78(37.0)$ & $61(26.3)$ & $<0.001$ \\
\hline Autoimmune disease/asthma and allergy & $166(29.0)$ & $39(30.5)$ & $63(29.7)$ & $64(27.6)$ & 0.81 \\
\hline
\end{tabular}


Table I (Continued).

\begin{tabular}{|c|c|c|c|c|c|}
\hline & Total & PSQ+HI* & PSQ+MI & PSQ+LI & \multirow[t]{2}{*}{$p$-value for $\chi^{2}$ IANOVA } \\
\hline & $n=657$ & $n=150$ & $n=248$ & $n=259$ & \\
\hline Hematology disease & $83(14.5)$ & $23(18.0)$ & $31(14.7)$ & $29(12.5)$ & 0.37 \\
\hline \multicolumn{6}{|l|}{ Psychiatric diagnosis (n, \%) } \\
\hline Bipolar affective disorder & $313(47.6)$ & $55(36.7)$ & $103(4 \mid .5)$ & $155(59.8)$ & $<0.001$ \\
\hline Major depressive disorder & $344(52.4)$ & $95(63.3)$ & $145(58.5)$ & $104(40.2)$ & \\
\hline \multicolumn{6}{|l|}{ Use of psychotropic agents (n, \%) } \\
\hline Antidepressants & $358(54.8)$ & $107(72.3)$ & 145 (58.9) & $106(40.9)$ & $<0.001$ \\
\hline Mood stabilizers & $239(36.5)$ & $39(26.2)$ & $78(31.7)$ & $122(47.1)$ & $<0.001$ \\
\hline Antipsychotics & $264(40.4)$ & 71 (47.7) & $94(38.2)$ & $99(38.2)$ & 0.12 \\
\hline Hypnotics use & $432(75.7)$ & III (88.I) & $163(76.5)$ & $158(68.1)$ & $<0.001$ \\
\hline $\begin{array}{l}\text { Beck Depression Inventory-II [mean } \\
\text { (SD)] }\end{array}$ & I4.8 (I I.8) & $31.8(5.1)$ & $16.3(6.1)$ & $3.5(2.9)$ & $<0.001$ \\
\hline Beck Anxiety Inventory [mean (SD)] & $13.2(12.2)$ & $26.1(11.4)$ & $13.3(12.2)$ & $6.1(7.4)$ & $<0.001$ \\
\hline
\end{tabular}

Abbreviations: *PSQ, poor sleep quality; HI, high insomnia; MI, moderate insomnia; LI, low insomnia; SD, standard deviation.

Table 2 Model Fitting Results for 3- to 5-Class Solutions in the Latent Class Analysis ( $n=657)$

\begin{tabular}{|l|c|c|c|}
\hline & 3 Classes & 4 Classes & 5 Classes \\
\hline Akaike Information Criterion & $20,533.92$ & $20,178.22$ & $20,025.83$ \\
Sample-Size Adjusted Bayesian Information Criterion & $20,694.07$ & $20,392.19$ & $20,293.62$ \\
Entropy & 0.93 & 0.91 & 0.91 \\
Lo-Mendell-Rubin Adjusted Likelihood Ratio test & 0.0046 & 0.0002 & 0.6234 \\
Parametric Bootstrapped Likelihood Ratio test & $<0.0001$ & $<0.0001$ & $<0.0001$ \\
\hline
\end{tabular}

Table 3 Univariate Analysis for Sleep-Wake-Related Disturbances in Subgroups $(n=657)$

\begin{tabular}{|c|c|c|c|c|c|}
\hline & Total & PSQ+HI* & PSQ+MI & PSQ+LI & \multirow[t]{2}{*}{$p$-value for $\chi 2 / A N O N A$} \\
\hline & $n=657$ & $n=150$ & $n=248$ & $n=259$ & \\
\hline \multicolumn{6}{|l|}{ Pittsburgh Sleep Quality Index } \\
\hline Total scores $\geq 6(n, \%)$ & $485(84.9)$ & 118 (93.7) & $189(88.7)$ & 178 (76.7) & $<0.001$ \\
\hline \multicolumn{6}{|l|}{ Component scores $\geq \mathrm{I}(\mathrm{n}, \%)$} \\
\hline Subjective sleep quality & $245(42.9)$ & $84(66.7)$ & $98(46.0)$ & $63(27.2)$ & $<0.001$ \\
\hline Sleep latency & $314(55.0)$ & $86(68.3)$ & $116(54.5)$ & $112(48.3)$ & 0.001 \\
\hline Sleep duration & $119(20.8)$ & $40(31.7)$ & $40(18.8)$ & $39(16.8)$ & 0.003 \\
\hline Sleep efficiency & $125(21.9)$ & $41(32.5)$ & $42(19.7)$ & $42(18.1)$ & 0.004 \\
\hline Sleep disturbance & $448(78.5)$ & $105(83.3)$ & $173(81.2)$ & $170(73.3)$ & 0.04 \\
\hline Daytime dysfunction & $162(28.4)$ & $63(50.0)$ & $61(28.6)$ & $38(16.4)$ & $<0.001$ \\
\hline Eveningness chronotype (n, \%) & $126(26.1)$ & $45(42.9)$ & $55(29.6)$ & $26(13.6)$ & $<0.001$ \\
\hline \multicolumn{6}{|c|}{ Night and daytime symptoms of insomnia } \\
\hline Subjective sleep loss $\geq 2$ hours & $177(34.3)$ & $49(44.5)$ & $70(36.8)$ & $58(26.9)$ & 0.004 \\
\hline Sleep initiation difficulty (n, \%) & $203(35.9)$ & $54(43.5)$ & $81(38.6)$ & $68(29.3)$ & 0.02 \\
\hline Sleep maintenance difficulty (n, \%) & $207(36.6)$ & $64(51.6)$ & $76(36.2)$ & $67(28.9)$ & $<0.001$ \\
\hline Early morning awakening (n, \%) & $171(30.3)$ & $52(41.9)$ & $58(25.1)$ & $61(29.0)$ & 0.004 \\
\hline Daytime sleepiness (n, \%) & $231(41.0)$ & $63(50.8)$ & $76(33.2)$ & $92(43.8)$ & 0.003 \\
\hline
\end{tabular}

Abbreviations: *PSQ, poor sleep quality; HI, high insomnia; MI, moderate insomnia; LI, low insomnia. 


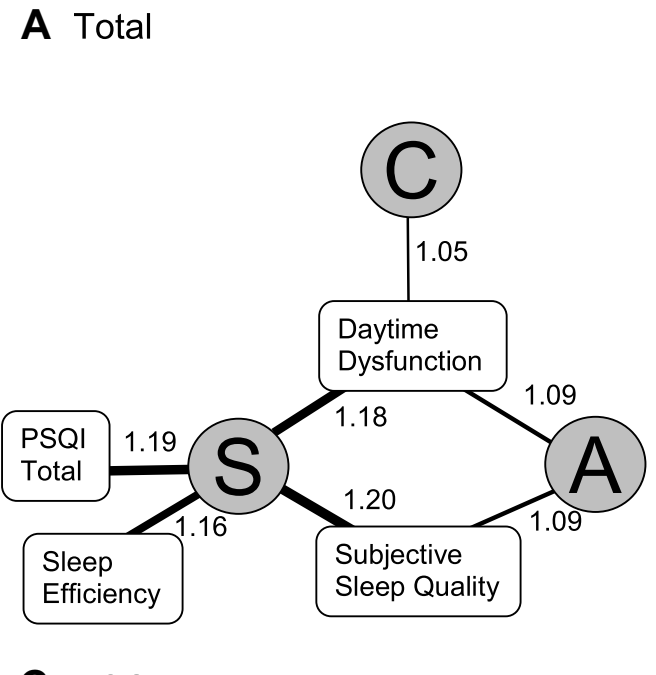

C $\mathrm{PSQ}+\mathrm{Ml}$

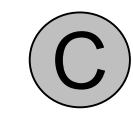

B PSQ+HI
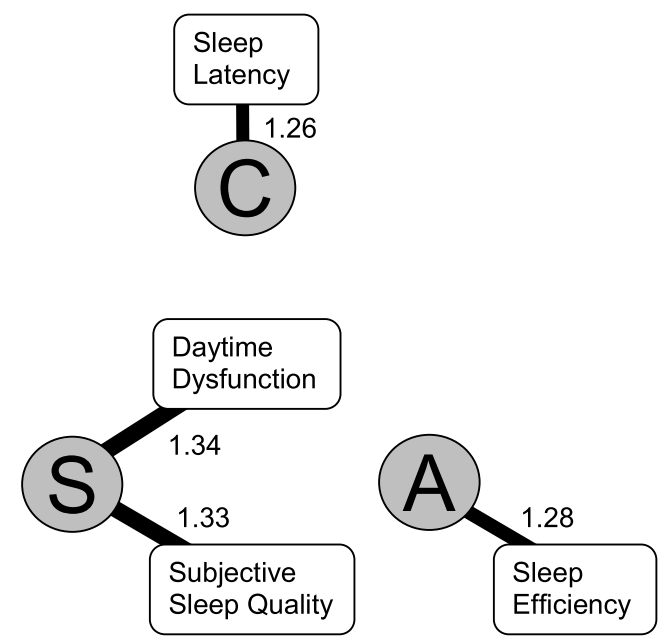

D $P S Q+L I$
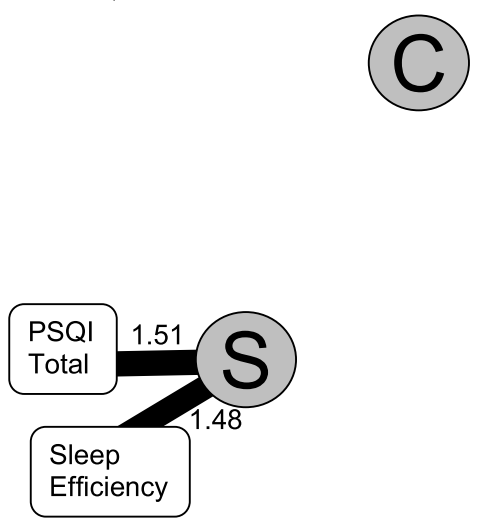

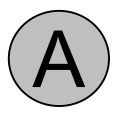

Figure I The co-occurring patterns between domains of depressive symptomatology with global scores and component scores in the Pittsburgh Sleep Quality Index (PSQI). C: cognitive domain; A: affective domain; S: somatic domain. The number (odds ratio) and thickness of the lines linking each domain and PSQI-related components reflect the effect sizes of associations. The co-occurring patterns are illustrated by participant groups. Panel (A): total participants. Panel (B): the poor sleep quality with high insomnia (PSQ+HI) group. Panel (C): the poor sleep quality with moderate insomnia (PSQ+MI) group. Panel (D): the poor sleep quality with low insomnia (PSQ+LI) group.

PSQI-defined poor sleep quality and six components in all participants and each subgroup. The results of multiple logistic regression analyses are detailed in Supplement Table S1. Of participants, daytime dysfunction was the only component related to all three domains of depression (panel A). In subgroup analyses, additional co-occurring patterns were observed in specific subgroups. In the PSQ $+\mathrm{HI}$ group, cognitive and affective domains were related to sleep latency and efficiency, respectively (panel B). Figure 2 depicts the independent relationship between domains of depressive symptomatology with chronotype preference and insomnia symptoms. The results of multiple logistic regression analyses are presented in Supplement Table S2. Cognitive and somatic domains were more likely to co-occur with evening chronotype, while the somatic domain was additionally related to subjective sleep loss (panel A). Among the subgroups, the
PSQ+HI group contained the highest number of cooccurring associations. Despite a null association when analyzing total participants, sleep initiation difficulties were specifically related to the cognitive domain in the PSQ+HI group (panel B) and to the affective domain in the PSQ+MI group (panel C). In the PSQ+HI group, the somatic domain was related to early morning awakening and daytime sleepiness (panel B); these associations were only identified in the subgroup analyses.

Integration of the identified co-occurring patterns revealed that depressive symptomatology exhibited various co-occurring patterns with SWRDs. In general, the somatic domain exhibited the highest number of associations with SWRDs. When comparing co-occurring patterns in each subgroup, a progressive order was noted (Figure S2). In the order of PSQ+LI, PSQ+MI, and PSQ+HI groups, the cooccurring associations between various SWRDs and 
A Total

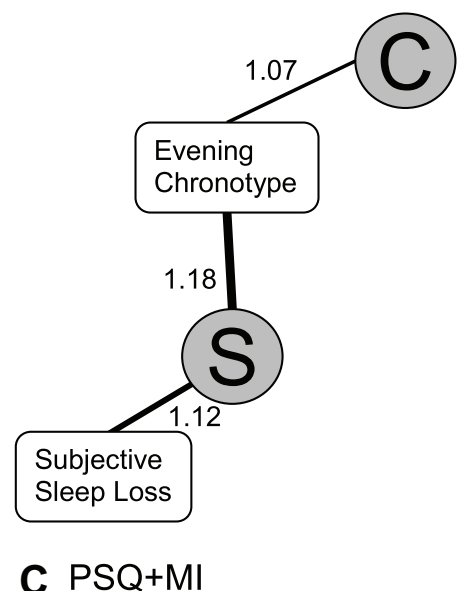

C PSQ+M
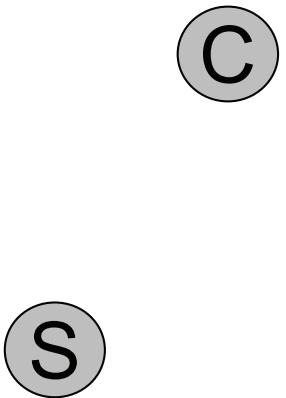
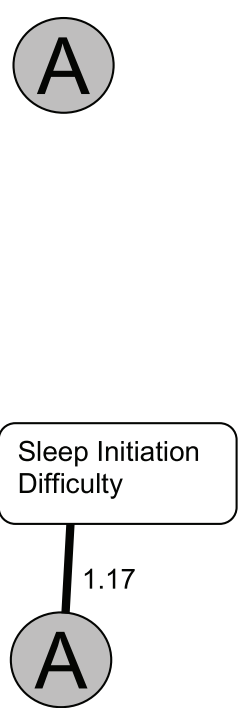

B PSQ+HI

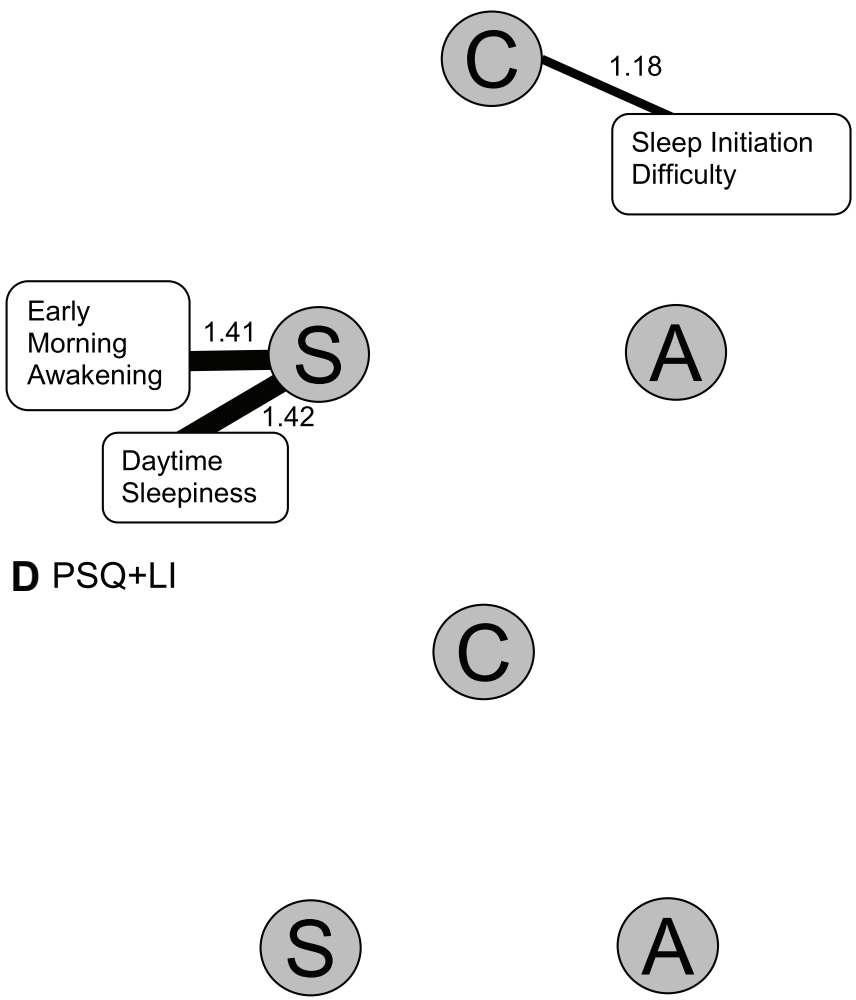

Figure 2 The co-occurring patterns between domains of depressive symptomatology with various nighttime and daytime symptoms of insomnia and chronotype preferences. C: cognitive domain; A: affective domain; S: somatic domain. The number (odds ratio) and thickness of the lines linking each domain and PSQI-related components reflect the effect sizes of associations. The co-occurring patterns are illustrated by participant groups. Panel (A): total participants. Panel (B): the poor sleep quality with high insomnia (PSQ+HI) group. Panel (C): the poor sleep quality with moderate insomnia (PSQ+MI) group. Panel (D): the poor sleep quality with low insomnia (PSQ+LI) group.

domains of depressive symptomatology appeared in the sequence of somatic, affective, and cognitive domains.

\section{Discussion}

This study aimed to elucidate the complex associations between depressive symptomatology and SWRDs in individuals with mood disorders. To this end, we categorized depressive symptomatology into three domains and further empirically subgrouped participants to disentangle the heterogeneity of depressive symptomatology between individuals. Our findings indicate that even in the non-acute state, a large proportion of participants still presented with SWRDs. In addition, the three domains of depression exhibited different co-occurring patterns with SWRDs, and these patterns further varied in empirically derived subgroups.

Although there is abundant literature on the heterogeneity of depression based on LCA,${ }^{40}$ to the best of our knowledge, the present study is the first to recruit only participants with a non-acute state of depression. In a systematic review that included 24 articles using LCA to identify depression subgroups, half of the articles were based on community populations, and the other half was based on clinical populations. ${ }^{40}$ Most of the clinical samples focused on the acute state of mood disorders, and the others examined cohorts comprising both acute and non-acute state patients. Compared to individuals in the acute state, those in the nonacute state are more likely to reflect long-term trait-like psychopathology which may indicate underlying neurophysiological pathomechanisms. Further, only one previous study that also used LCA to subgroup depression addressed the associations between depression symptoms with night symptoms of insomnia. ${ }^{41}$ Thus, the analytical strategy, characteristics of participants, and various types of SWRDs are unique features of the present study.

\section{Rationale for Classifying BDI-II Depressive Symptoms}

In the present study, depressive symptoms of the BDI-II were empirically assigned to three domains. This conceptual 
approach is similar to that proposed by the developer of the BDI-II, in which somatic-affective and cognitive factors were conceptualized. ${ }^{42}$ In the literature, the factor structure of the BDI-II has been investigated in different populations, and the number of factors remains controversial. In general, cognitive, affective, and somatic domains are the three most commonly proposed factors; these three factors are occasionally reduced to two factors. ${ }^{25,26}$ In this study, "concentration difficulty" was categorized as a cognitive domain symptom, which differed from previous studies. Since concentration difficulty and indecisiveness have been categorized into the same factor ${ }^{43,44}$ and are classified as a single diagnostic criterion in the DSM system, we employed this strategy to increase the clinical relevance of our study.

\section{Differential Co-Occurring Patterns Between Domains of Depressive Symptomatology and SWRDs}

Of the three domains, the somatic domain was most likely to be associated with various nighttime symptoms of SWRDs. This finding agrees with previous reports of factor analysis indicating that sleep-related items were almost always classified into similar factors as symptoms of loss of energy, change in appetite, or interest in sex. ${ }^{25}$ This finding also suggests a shared pathomechanism involving the hypothalamus, which maintains fundamental physiological demands, such as sleep, appetite, and sex. ${ }^{45}$

In contrast to previous research on nighttime symptoms of SWRDs and mood disorders, the present study highlighted the importance of addressing daytime manifestations of SWRDs. In this study, the prevalence of daytime sleepiness, which was defined as daytime symptoms of insomnia, was high in all participants (41\%) and higher than that in the general population $(1.0-6.4 \%){ }^{46}$ Furthermore, PSQI-defined daytime dysfunction cooccurred with all domains of depression in all participants. This consistent co-occurring pattern across domains echoes previous arguments that daytime symptoms of insomnia are more strongly correlated with depressive symptomatology when compared to nighttime symptoms. ${ }^{47,48}$ Daytime SWRDs may indicate residual symptoms of depression, suboptimal treatment of SWRDs, or adverse effects from psychotropic agents. Further investigations of the prevalence of daytime SWRDs in individuals with non-acute mood disorders are warranted.
Another notable finding of the present study was the co-occurring pattern of the eveningness chronotype and the cognitive and somatic domains of depressive symptomatology. This finding is consistent with previous reports that inpatients with mood disorders and eveningness preference had higher scores in the cognitive and somatic domains of the BDI-II. ${ }^{49}$ Several mechanisms may underpin the link between the cognitive domain and eveningness preference. First, the eveningness chronotype is associated with suicidal behavior. ${ }^{50}$ Second, the CLOCK gene rs $1801260 \mathrm{C}$ allele is correlated with both eveningness preference $^{51}$ and hopelessness, ${ }^{52}$ which is a psychopathology under the umbrella of pessimism. Since both suicidal ideation and pessimism are cognitive symptoms of the BDI-II, our findings suggest a similar phenomenon.

In the present study, the somatic domain included four items of the BDI-II including loss of energy, changes in appetite, tiredness or fatigue, and loss of interest in sex. These symptoms are at least partly controlled by the arousal system and/or reward system in the central nervous system. The neural substrate underpinning the cooccurring evening preference and somatic domain of depressive symptomatology may be the hypothalamus, which regulates circadian rhythmicity and modulates arousal and reward systems. ${ }^{53-55}$ In addition, this co-occurring pattern may also be due to a misalignment between inner circadian rhythms and external social rhythms. ${ }^{56,57}$

\section{Differential Co-Occurring Patterns Between Depressive Symptomatology and SWRDs Among Subgroups}

In this study, we used LCA to identify subgroups with homogeneous profiles of the BDI-II symptomatology. Among the three subgroups, differential co-occurring patterns were observed. This finding suggests distinct pathomechanisms and clinical implications. First, in the order of PSQ $+\mathrm{LI}, \mathrm{PSQ}+\mathrm{MI}$, and PSQ+HI, the corresponding sequence of specific domains of depressive symptomatology cooccurring with any SWRD was somatic, affective, and cognitive. As these three subgroups differed in the overall severity of depressive symptoms, our findings suggest that in some subgroups of patients, cognitive therapy for both SWRDs and depressive symptoms may be beneficial, such as in the PSQ+HI group. In contrast, somatic or behavioral treatments may be the most effective intervention in other circumstances, such as in the PSQ+LI group. Second, the 
co-occurring patterns that were only identified in subgroup analysis warrant further attention. For example, sleep initiation difficulties and PSQI-defined prolonged sleep latency co-occurred with specific domains in the subgroup analyses but not in analyses that used the entire sample. Statistically, co-occurring patterns that were identified using the total sample but failed to appear in subsequent stratified analyses could be interpreted as inadequate statistical power in each subgroup analysis. In contrast, the identification of cooccurring patterns only in specific subgroups suggested that differential strength of co-occurring patterns between subgroups nullifies an association that should appear in a specific subgroup. For example, the co-occurring pattern for depressive domains with sleep initiation difficulties was uniquely identified in the PSQ+HI and PSQ+HI subgroups. Notably, sleep initiation difficulties co-occurred with the cognitive domain in the PSQ $+\mathrm{HI}$ subgroup and with the affective domain in the PSQ+MI subgroup. This groupspecific association hints at different pathomechanisms underlying similar SWRD phenotypes in individuals with non-acute state mood disorders. As insomnia is formulated as a consequence of hyperarousal (cognitive, affective, and somatic) in modern insomnia theoretical models, ${ }^{58,59}$ cognition-related hyperarousal in the PSQ+HI group and affectrelated hyperarousal in the PSQ + LI group may differentially explain an identical insomnia phenotype that requires distinct interventions.

\section{Limitations}

This study has several limitations. First, this was a crosssectional study; therefore, causal inferences could not be drawn. Second, multiple comparisons in a series of logistic regression analyses may have inflated type I errors. Furthermore, psychotropic agents other than hypnotics may have introduced additional confounding effects. Third, participants in this study were from mixed samples of MDD and BAD, for which the pathomechanisms for depressive symptomatology may differ in essence. However, we controlled for psychiatric diagnosis in the multiple logistic regression analyses. Therefore, the potential confound from diagnosis was at least partly mitigated. Fourth, this study did not include a healthy control group, which may provide additional insight into the clinical implications of the results. Finally, although depressive symptoms in the BDI-II were categorized into three domains, it remains unclear whether all symptoms in a specific domain could be ascribed to a common neurobiological pathomechanism regardless of whether the classification was performed empirically or by factor analysis.

\section{Conclusions}

SWRDs provide an important window into the biological basis of depressive symptoms. Our findings support the arguments of diverse neurophysiological pathogenesis in depressive disorders and hint at neurophysiological targets in specific subgroups of patients in terms of investigation of pathomechanisms or clinical interventions. The new paradigm of "co-occurrence/co-existence" has been proposed to replace traditional primary-secondary concepts in most recent diagnostic systems to conceptualize co-occurring depressive disorders with insomnia disorders. In parallel, the clinical guidelines for insomnia inform clinicians regarding the simultaneous management of insomnia and co-occurring morbidities. However, tailored interventions for individuals with these co-occurring conditions are lacking. The present study suggests a novel strategy to disentangle heterogeneous etiologies in individuals with depressive disorder. In the future, studies using functional neuroimaging or interventions that target specific brain areas are necessary to validate the shared biological substrates for these co-occurring patterns.

\section{Abbreviations}

AIC, Akaike's information criterion; AOR, adjusted odds ratio; BAD, bipolar affective disorder; BAI, Beck Anxiety Inventory; BDI-II, Beck Depression Inventory II; DSM, Diagnostic and Statistical Manual of Mental Disorders; LCA, latent class analysis; MDD, major depressive disorder; MEQ, Morningness-Eveningness questionnaire; PSQI, Pittsburgh Sleep Quality Index; PSQ+HI, poor sleep quality and high insomnia; PSQ+LI, poor sleep quality and low insomnia; PSQ+MI, poor sleep quality and moderate insomnia; REM, rapid eye movement; SADS-L, Schedule for Affective Disorder and Schizophrenia-Lifetime; SD, standard deviation; sBIC, sample-size adjusted Bayesian's information criterion; SWRD, Sleep-wake-related disturbances.

\section{Data Sharing Statement}

All data and materials related to the study can be obtained by contacting the corresponding author.

\section{Author Contributions}

All authors made a significant contribution to the work reported, whether that is in the conception, study design, execution, acquisition of data, analysis and interpretation, 
or in all these areas; took part in drafting, revising or critically reviewing the article; gave final approval of the version to be published; have agreed on the journal to which the article has been submitted; and agree to be accountable for all aspects of the work.

\section{Funding}

Participants recruitment was supported by National Taiwan University Hospital grant (UN110-021), Taiwan Ministry of Science and Technology (MOST) grants (105-2628-B-002-028-MY3, 108-2314-B-002-110-MY2, and 108-2314-B-002-136-MY3) and a grant from National Health Research Institute (NHRI-EX108 $-10627 \mathrm{NI})$. The sponsor has no role in any of the stages from study design to submission of the paper for publication.

\section{Disclosure}

Financial competing interests: none.

Non-financial competing interests: none.

\section{References}

1. GBD 2017 Disease and Injury Incidence and Prevalence Collaborators. Global, regional, and national incidence, prevalence, and years lived with disability for 354 diseases and injuries for 195 countries and territories, 1990-2017: a systematic analysis for the Global Burden of Disease Study 2017. Lancet. 2018;392 (10159):1789-1858.

2. Post RM, Denicoff KD, Leverich GS, et al. Morbidity in 258 bipolar outpatients followed for 1 year with daily prospective ratings on the NIMH life chart method. J Clin Psychiatry. 2003;64(6):680-690. doi:10.4088/JCP.v64n0610

3. Gao K, Su M, Sweet J, Calabrese JR. Correlation between depression/ anxiety symptom severity and quality of life in patients with major depressive disorder or bipolar disorder. $J$ Affect Disord. 2019;244:9-15. doi:10.1016/j.jad.2018.09.063

4. Geoffroy PA, Hoertel N, Etain B, et al. Insomnia and hypersomnia in major depressive episode: prevalence, sociodemographic characteristics and psychiatric comorbidity in a population-based study. $J$ Affect Disord. 2018;226:132-141. doi:10.1016/j.jad.2017.09.032

5. Palagini L, Baglioni C, Ciapparelli A, Gemignani A, Riemann D. REM sleep dysregulation in depression: state of the art. Sleep Med Rev. 2013;17(5):377-390. doi:10.1016/j.smrv.2012.11.001

6. Jansson-Frojmark M, Lindblom K. A bidirectional relationship between anxiety and depression, and insomnia? A prospective study in the general population. J Psychosom Res. 2008;64(4):443-449. doi:10.1016/j.jpsychores.2007.10.016

7. Dombrovski AY, Mulsant BH, Houck PR, et al. Residual symptoms and recurrence during maintenance treatment of late-life depression. J Affect Disord. 2007;103(1-3):77-82. doi:10.1016/j.jad.2007.01.020

8. Dombrovski AY, Cyranowski JM, Mulsant BH, et al. Which symptoms predict recurrence of depression in women treated with maintenance interpersonal psychotherapy? Depression and Anxiety. 2008;25 (12):1060-1066. doi:10.1002/da.20467

9. Sivertsen B, Salo P, Mykletun A, et al. The bidirectional association between depression and insomnia: the HUNT study. Psychosom Med. 2012;74(7):758-765. doi:10.1097/PSY.0b013e3182648619
10. Krishnan V, Nestler EJ. Linking molecules to mood: new insight into the biology of depression. Am $J$ Psychiatry. 2010;167 (11):1305-1320. doi:10.1176/appi.ajp.2009.10030434

11. Joiner WJ. The Neurobiological Basis of Sleep and Sleep Disorders. Physiology. 2018;33(5):317-327. doi:10.1152/physiol.00013.2018

12. Saper CB, Cano G, Scammell TE. Homeostatic, circadian, and emotional regulation of sleep. J Comp Neurol. 2005;493(1):92-98. doi:10.1002/cne. 20770

13. Gehrman PR, Meltzer LJ, Moore M, et al. Heritability of insomnia symptoms in youth and their relationship to depression and anxiety. Sleep. 2011;34(12):1641-1646. doi:10.5665/sleep.1424

14. Landolt HP, Kelsoe JR, Rapaport MH, Gillin JC. Rapid tryptophan depletion reverses phenelzine-induced suppression of REM sleep. J Sleep Res. 2003;12(1):13-18. doi:10.1046/j.1365-2869.2003.00336.x

15. McCall WV, Rosenquist PB. Letter to the editor: the effect of ECT on sleep-a comment to Winkler et al. J Psychiatr Res. 2015;61:239-240. doi:10.1016/j.jpsychires.2014.10.008

16. Fried EI. The 52 symptoms of major depression: lack of content overlap among seven common depression scales. J Affect Disord. 2017;208:191-197. doi:10.1016/j.jad.2016.10.019

17. Fried EI, von Stockert S, Haslbeck JMB, Lamers F, Schoevers RA, Penninx BWJH. Using network analysis to examine links between individual depressive symptoms, inflammatory markers, and covariates. Psychol Med. 2019;1-9.

18. Fried EI, Nesse RM. Depression is not a consistent syndrome: an investigation of unique symptom patterns in the STAR*D study. $J$ Affect Disord. 2015;172:96-102. doi:10.1016/j.jad.2014.10.010

19. Fried EI. Moving forward: how depression heterogeneity hinders progress in treatment and research. Expert Rev Neurother. 2017;17 (5):423-425. doi:10.1080/14737175.2017.1307737

20. Zimmerman M, Martinez JH, Young D, Chelminski I, Dalrymple K. Severity classification on the Hamilton Depression Rating Scale. J Affect Disord. 2013;150(2):384-388. doi:10.1016/j.jad.2013.04.028

21. Young RC, Fau-Biggs JT, Biggs JT, Meyer DA. A rating scale for mania: reliability, validity and sensitivity. Br J Psychiatry. 1978;133 (5):429-435. doi:10.1192/bjp.133.5.429

22. Endicott J, Spitzer RL. A diagnostic interview: the schedule for affective disorders and schizophrenia. Arch Gen Psychiatry. 1978;35(7):837-844. doi:10.1001/archpsyc.1978.01770310043002

23. Huang SY, Lin WW, Ko HC, et al. Possible interaction of alcohol dehydrogenase and aldehyde dehydrogenase genes with the dopamine D2 receptor gene in anxiety-depressive alcohol dependence. Alcohol Clin Exp Res. 2004;28(3):374-384. doi:10.1097/01. ALC.0000117832.62901.61

24. Lu ML, Che HH, Chang SW, Shen WW. Reliability and Validity of the Chinese Version of the Beck Depression Inventory-II. Taiwanese J Psychiatry. 2002;16(4):301-310.

25. Vanheule S, Desmet M, Groenvynck H, Rosseel Y, Fontaine J. The factor structure of the Beck Depression Inventory-II: an evaluation. Assessment. 2008;15(2):177-187. doi:10.1177/1073191107311261

26. Manian N, Schmidt E, Bornstein MH, Martinez P. Factor structure and clinical utility of BDI-II factor scores in postpartum women. $J$ Affect Disord. 2013;149(1-3):259-268. doi:10.1016/j.jad.2013.01.039

27. Garcia-Batista ZE, Guerra-Pena K, Cano-Vindel A, HerreraMartinez SX, Medrano LA. Validity and reliability of the Beck Depression Inventory (BDI-II) in general and hospital population of Dominican Republic. PLoS One. 2018;13(6):e0199750. doi:10.1371/ journal.pone. 0199750

28. Buysse DJ, Reynolds CF, Monk TH, Berman SR, Kupfer DJ. The Pittsburgh Sleep Quality Index: a new instrument for psychiatric practice and research. Psychiatry Res. 1989;28(2):193-213. doi:10.1016/0165-1781(89)90047-4

29. Tsai PS, Wang SY, Wang MY, et al. Psychometric evaluation of the Chinese version of the Pittsburgh Sleep Quality Index (CPSQI) in primary insomnia and control subjects. Quality Life Res. 2005;14 (8):1943-1952. doi:10.1007/s11136-005-4346-x 
30. Smith CS, Reilly C, Midkiff K. Evaluation of three circadian rhythm questionnaires with suggestions for an improved measure of morningness. J Appl Psychol. 1989;74(5):728-738. doi:10.1037/ 0021-9010.74.5.728

31. Gau SS, Merikangas KR. Similarities and differences in sleep-wake patterns among adults and their children. Sleep. 2004;27(2):299-304. doi:10.1093/sleep/27.2.299

32. Che -H-H, Lu M-L, Chen H-C, Chang S-W, Lee Y-J. Validation of the Chinese Version of the Beck Anxiety Inventory. Formosan J Med. 2006;10(4):447-454.

33. Beck AT, Epstein N, Brown G, Steer RA. An inventory for measuring clinical anxiety: psychometric properties. J Consult Clin Psychol. 1988;56(6):893-897. doi:10.1037/0022-006X.56.6.893

34. Muthén LK, Muthén BO. Mplus User's Guide. Seventh ed. Los Angeles: CA: Muthén \& Muthén; 2015.

35. Akaike H. Factor analysis and AIC. Psychometrika. 1987;52 (3):317-332. doi:10.1007/BF02294359

36. Sclove SL. Application of model-selection criteria to some problems in multivariate analysis. Psychometrika. 1987;52(3):333-343. doi:10.1007/BF02294360

37. Celeux G, Soromenho G. An entropy criterion for assessing the number of clusters in a mixture model. J Classification. 1996;13 (2):195-212. doi:10.1007/BF01246098

38. Roeder K, Lynch KG, Nagin DS. Modeling Uncertainty in Latent Class Membership: a Case Study in Criminology. J Am Stat Assoc. 1999;94(447):766-776. doi:10.1080/01621459.1999.10474179

39. Tsai HC, Lu MK, Yang YK, et al. Empirically derived subgroups of bipolar I patients with different comorbidity patterns of anxiety and substance use disorders in Han Chinese population. J Affect Disord. 2012;136(1-2):81-89. doi:10.1016/j.jad.2011.08.015

40. Ulbricht CM, Chrysanthopoulou SA, Levin L, Lapane KL. The use of latent class analysis for identifying subtypes of depression: a systematic review. Psychiatry Res. 2018;266:228-246. doi:10.1016/j.psychres.2018.03.003

41. Ulbricht CM, Rothschild AJ, Lapane KL. The association between latent depression subtypes and remission after treatment with citalopram: a latent class analysis with distal outcome. J Affect Disord. 2015;188:270-277. doi:10.1016/j.jad.2015.08.039

42. Beck AT, Steer RA, Brown GK. Manual for the Beck Depression Inventory-II. San Antonio: Psychological Corporation; 1996.

43. Osman A, Downs WR, Barrios FX, Kopper BA, Gutierrez PM, Chiros CE. Factor structure and psychometric characteristics of the beck depression inventory-II. J Psychopathol Behav Assess. 1997;19 (4):359-376. doi:10.1007/BF02229026

44. Byrne BM, Stewart SM, Lee PWH. Validating the Beck Depression Inventory-II for Hong Kong Community Adolescents. Int J Testing. 2004;4(3):199-216. doi:10.1207/s15327574ijt0403_1

45. Vanderah TW, Gould DJ. Nolte's the Human Brain: An Introduction to Its Functional Anatomy. Eighth ed. ed, Elsevier; 2021.
46. Kao CC, Huang CJ, Wang MY, Tsai PS. Insomnia: prevalence and its impact on excessive daytime sleepiness and psychological well-being in the adult Taiwanese population. Quality Life Res. 2008;17 (8):1073-1080. doi:10.1007/s11136-008-9383-9

47. Koffel E, Watson D. The two-factor structure of sleep complaints and its relation to depression and anxiety. J Abnorm Psychol. 2009;118 (1):183-194. doi:10.1037/a0013945

48. Lai H-C, Hsu N-W, Chou P, Chen H-C. The associations between various sleep-wake disturbances and depression in community-dwelling older adults- the Yilan study, Taiwan. Aging Ment Health. 2020;24 (5):717-724. doi:10.1080/13607863.2019.1582006

49. Muller MJ, Olschinski C, Kundermann B, Cabanel N. Patterns of self-reported depressive symptoms in relation to morningness-eveningness in inpatients with a depressive disorder. Psychiatry Res. 2016;239:163-168. doi:10.1016/j.psychres.2016.03.018

50. Rumble ME, Dickson D, McCall WV, et al. The relationship of person-specific eveningness chronotype, greater seasonality, and less rhythmicity to suicidal behavior: a literature review. $J$ Affect Disord. 2018;227:721-730. doi:10.1016/j.jad.2017.11.078

51. Katzenberg D, Young T, Finn L, et al. A CLOCK polymorphism associated with human diurnal preference. Sleep. 1998;21 (6):569-576. doi:10.1093/sleep/21.6.569

52. Suzuki M, Dallaspezia S, Locatelli C, et al. CLOCK gene variants associated with the discrepancy between subjective and objective severity in bipolar depression. J Affect Disord. 2017;210:14-18. doi:10.1016/j.jad.2016.12.007

53. Harrington ME. Neurobiological studies of fatigue. Prog Neurobiol. 2012;99(2):93-105. doi:10.1016/j.pneurobio.2012.07.004

54. Parekh PK, McClung CA. Circadian Mechanisms Underlying Reward-Related Neurophysiology and Synaptic Plasticity. Front Psychiatry. 2015;6:187. doi:10.3389/fpsyt.2015.00187

55. Aston-Jones G, Smith RJ, Moorman DE, Richardson KA. Role of lateral hypothalamic orexin neurons in reward processing and addiction. Neuropharmacology. 2009;56(Suppl 1):112-121. doi:10.1016/j.neuropharm.2008.06.060

56. Levandovski R, Dantas G, Fernandes LC, et al. Depression scores associate with chronotype and social jetlag in a rural population. Chronobiol Int. 2011;28(9):771-778. doi:10.3109/07420528.2011.602445

57. Wittmann M, Dinich J, Merrow M, Roenneberg T. Social jetlag: misalignment of biological and social time. Chronobiol Int. 2006;23 (1-2):497-509. doi:10.1080/07420520500545979

58. Lundh LG, Broman JE. Insomnia as an interaction between sleep-interfering and sleep-interpreting processes. J Psychosom Res. 2000;49(5):299-310. doi:10.1016/S0022-3999(00)00150-1

59. Perlis ML, Giles DE, Mendelson WB, Bootzin RR, Wyatt JK. Psychophysiological insomnia: the behavioural model and a neurocognitive perspective. J Sleep Res. 1997;6(3):179-188. doi:10.1046/j.1365-2869.1997.00045.x
Nature and Science of Sleep

\section{Publish your work in this journal}

Nature and Science of Sleep is an international, peer-reviewed, open access journal covering all aspects of sleep science and sleep medicine, including the neurophysiology and functions of sleep, the genetics of sleep, sleep and society, biological rhythms, dreaming, sleep disorders and therapy, and strategies to optimize healthy sleep.
The manuscript management system is completely online and includes a very quick and fair peer-review system, which is all easy to use. Visit http://www.dovepress.com/testimonials.php to read real quotes from published authors. 